(6) OPEN ACCESS

\title{
Intimate partner violence against low-income women in Mexico City and associations with work-related disruptions: a latent class analysis using cross- sectional data
}

\author{
Jhumka Gupta, ${ }^{1}$ Tiara C Willie, ${ }^{2,3}$ Courtney Harris, ${ }^{1}$ Paola Abril Campos, ${ }^{4}$ \\ Kathryn L Falb, ${ }^{5}$ Claudia Garcia Moreno, ${ }^{6}$ Claudia Diaz Olavarrieta, \\ Cassandra A Okechukwu ${ }^{4}$
}

- Additional material is published online only. To view please visit the journal online (http://dx.doi.org/10.1136/ jech-2017-209681)

'Department of Global and Community Health, College of Health and Human Services, George Mason University, Fairfax, VA, USA

${ }^{2}$ Center for Interdisciplinary Research on AIDS, Yale University, New Haven,

Connecticut, USA

${ }^{3}$ Department of Social and Behavioral Sciences, Yale School of Public Health, New Haven, Connecticut, USA

${ }^{4}$ Department of Social and Behavioral Sciences, Harvard T.H. Chan School of Public Health, Boston, Massachusetts, USA ${ }^{5}$ International Rescue Committee, New York City, New York, USA

${ }^{6}$ Department of Reproductive Health and Research, World Health Organization, Geneva, Switzerland

${ }^{7}$ Population Council of Mexico, Mexico City, Mexico

Correspondence to Dr Jhumka Gupta, Department of Global and Community Health, College of Health and Human Services, George Mason University, Fairfax, VA 22030 USA; jgupta4@gmu.edu

Received 5 July 2017

Revised 6 February 2018 Accepted 15 February 2018

Published Online First

7 March 2018

\begin{abstract}
Background Disrupting women's employment is a strategy that abusive partners could use to prevent women from maintaining economic independence and stability. Yet, few studies have investigated disruptions in employment among victims of intimate partner violence (IPV) in low-income and middle-income countries. Moreover, even fewer have sought to identify which female victims of IPV are most vulnerable to such disruptions.

Methods Using baseline data from 947 women in Mexico City enrolled in a randomised controlled trial, multilevel latent class analysis (LCA) was used to classify women based on their reported IPV experiences. Furthermore, multilevel logistic regression analyses were performed on a subsample of women reporting current work $(n=572)$ to investigate associations between LCA membership and IPV-related employment disruptions. Results Overall, $40.6 \%$ of women who were working at the time of the survey reported some form of workrelated disruption due to IPV. LCA identified four distinct classes of IPV experiences: Low Physical and Sexual Violence (39.1\%); High Sexual and Low Physical Violence class (9.6\%); High Physical and Low Sexual Violence and Injuries (36.5\%); High Physical and Sexual Violence and Injuries (14.8\%). Compared with women in the Low Physical and Sexual Violence class, women in the High Physical and Sexual Violence and Injuries class and women in the High Physical and Low Sexual Violence and Injuries class were at greater risk of work disruption (adjusted relative risk (ARR) 2.44, 95\% Cl 1.80 to 3.29; ARR 2.05, 95\% CI 1.56 to 2.70, respectively). No other statistically significant associations emerged. Conclusion IPV, and specific patterns of IPV experiences, must be considered both in work settings and, more broadly, by economic development programmes.

Trial registration number NCT01661504.
\end{abstract}

\section{BACKGROUND}

Male-perpetrated intimate partner violence (IPV) against women is a pervasive global health issue with nearly one in three women experiencing such violence in their lifetime. ${ }^{1}$ In addition to impacting health outcomes (eg, reproductive and mental health), IPV adversely effects other important global development domains. ${ }^{1}$ Though research is skewed towards samples from the USA and other high-income countries, studies increasingly demonstrate that IPV has negative economic consequences for women's employment outcomes (eg, difficulties with finding and sustaining employment, work productivity) among women with IPV experiences. $^{2-5}$ This may be due to abusive partners' interference with women's employment through harassment and other job interference tactics. ${ }^{5}$ Moreover, one national US-based study of women's workplace homicides identified IPV as the second major cause of such deaths. ${ }^{6}$

To date, research on IPV's impacts on women's employment and other income-generating activities within low-income and middle-income countries (LMICs) is scarce. Much existing research pertaining to women's employment and IPV in LMICs is framed around women's employment status as being a risk factor for IPV, and IPV as a barrier to help-seeking and/or escaping an abusive partner. ${ }^{7-10}$ Less is known about how IPV may impact women's employment. One Nigeria-based study with women employed in the communications, trade, teaching and civil service industries found that IPV was significantly related to decreased work productivity. ${ }^{11}$ The extent to which these associations extend to lower-income women in other LMICs is currently unclear.

Understanding the impact of IPV on women's employment outcomes in Mexico is warranted. Although Mexico made progress towards its indicators for the Millennium Development Goals pertaining to gender and women's empowerment, a national survey documented IPV to be at $33 \% .^{12}$ Moreover, national data found $37.8 \%$ of economically active Mexican women reported some form of past-year IPV, ${ }^{12}$ and higher experiences of severe violence have been documented among women who are employed outside the home. ${ }^{13-15}$ The investigation of the impact of IPV on employment among lower- income women in Mexico is particularly critical, given limited financial resources and greater dependency on abusive partners. ${ }^{12}$ Furthermore, $51 \%$ of the informal self-employed workers are women. ${ }^{16}$

Furthermore, even less is known about which groups of women may be at greatest risk for 
employment disruptions. For instance, women who experience higher severity of IPV are more likely to face revictimisation, injuries and poorer health. ${ }^{17-19}$ These potential effects may make employment maintenance difficult. Growing US-based research has used person-centred approaches such as latent class analysis (LCA), to identify subgroups of women based on their patterns of IPV experiences, and relations to select health outcomes and resource use. ${ }^{20-23}$ Research using LCA with samples from LMICs is also emerging. ${ }^{24}$ Identifying groups of women via LCA may help inform development of targeted interventions. Achieving gender equality by providing equal access to decent work are key targets for the 2030 Sustainable Development Goals, ${ }^{25}$ but some subgroups of women based on IPV experiences may be at a greater disadvantage to realise targets.

The current study uses baseline data from a nurse-delivered randomised controlled trial with IPV survivors presenting to community health centres in Mexico City $^{26}$ to conduct the following aims: (1) use LCA to identify distinct groups of women based on past-year physical and sexual IPV experiences, and (2) assess associations between LCAs with disruptions in paid work due to IPV.

\section{METHODS}

The study used baseline data from a completed randomised controlled trial (RCT) with 42 community health clinics serving low-income women in Mexico City. Details on the trial and study protocols are detailed elsewhere (see online supplementary file of RCT findings and protocol paper) ${ }^{2627}$ and are briefly summarised. The RCT aimed to assess the impact of a nurse-delivered intervention to women identified as experiencing recent IPV through screening within public health clinics in Mexico City. All study protocols were approved by Yale School of Public Health (no. 1202007993), George Mason University (protocol no. 704016-4), Innovations for Poverty Action (no. 00006083) and the Mexico City Ministry of Health (protocol no. 14706812) human subjects committees.

\section{Study setting}

The study took place in Mexico City, the capital and most populous urban centre where almost half of the population is uninsured (approximately 3859963 residents). ${ }^{28}$ The uninsured population receives healthcare through a federal healthcare programme at Ministry of Health $(\mathrm{MoH})$ clinics in Mexico City. The Mexico City MoH health system comprises 206 health clinics (types I, II and III) and 31 hospitals. Type III clinics, the clinics selected for the RCT, offer more services and have a greater patient volume.

\section{Participants}

Eligible women were ages 18-44 years, in a current heterosexual relationship, responded in affirmative to past-year sexual or physical IPV, and were not pregnant or pregnant in their first trimester. Study exclusion criteria included cognitive impairment, seeking treatment for life-threatening emergency care or intending to relocate within 2 years. Trained female research assistants approached women in clinic waiting rooms, verified study eligibility and asked women to take an assessment screening that contained items from an abuse assessment screen that is widely used by International Planned Parenthood Federation/Western Hemisphere Region in Latin American and Caribbean countries including Mexico. ${ }^{29}{ }^{30}$ Based on piloting feedback, the final tool consisted of 11 questions in order to build rapport between the research assistant and participant (see online supplementary material for full assessment tool). ${ }^{27}$ If a woman answered at least one affirmative response to the validated physical and/or sexual IPV items, they were invited to participate in the study; those interested completed written and/ or verbal informed consent. Both the assessment and informed consent process took place within a private area of the clinic. A total of 29947 women presenting at the participating 42 clinics were approached and screened for eligibility. The baseline participation rate was $83.6 \%$ among women who could be determined to be eligible, for a total of 950 participants. ${ }^{26}$ Three additional women were excluded from the current analytic sample due to incomplete responses to all IPV items, yielding 947 women.

\section{Measures}

\section{Paid work disruption}

To assess frequency of past-year work disruptions due to pastyear IPV, participants were asked: "As a result of the abuse have you lost your job?"; "As a result of the abuse have you missed work?"; and "As a result of the abuse did you have to change jobs or schools?" A binary variable was then created (yes to any versus no to all).

\section{Latent class indicators of IPV experiences}

Past-year physical and/or sexual IPV and injuries were reported by women. IPV and injuries were assessed using items from the WHO Multi-Country study on Women's Health and Domestic Violence. ${ }^{31}$ Women were asked whether they experience various acts of physical and sexual violence by the male partners and if injuries occurred as a result of the violence. Past-year physical IPV was assessed by whether a woman reported whether her male partner had done the following: slapped her or thrown something at her that could hurt her; pushed, shoved, kicked or dragged her; choked her, strangled her or burned her intentionally; threatened to use a gun, knife or other weapon against her; and used a gun, knife or other weapon against her in the past year. Past-year sexual IPV was assessed by whether a woman reported her male partner had forced to have sex because of threats or intimidation or physically forced to have sex by their intimate partner when she did not want to. Injuries were assessed by whether a woman reported experiencing the following due to past year IPV: cuts that required stitches, a broken bone, lost tooth, burns, bruises, passed out or lost consciousness, and aches and pains. An affirmative response was coded as experiencing that specific past-year physical and/or sexual IPV behaviour and injury, while 'no' to all items was coded as no IPV. A binary variable was created for each specific form of IPV and injury.

\section{Demographics and work status}

Women were asked to report demographics such as age, number of children, birthplace, occupation, prior screening for IPV in the health sector, relationship status and reason for healthcare visit. A 'work status' variable was derived from three questions. First, we assessed women's involvement in the formal sector by asking women what their main occupation was with response options as 'Employee, Student, Housewife, Looking for Work, Own Personal Business'. Second, we assessed women's involvement in the informal sector by asking women, in addition to their main occupation, whether they do the following activities: 'Helping Family Business, Selling or Making Products, Animal Husbandry, Student, Employed in Other Sectors, or Other Activities (like ironing, car care, washing)'. Third, women were asked if they received any money or income for the activities they mentioned before. Women who said 'Yes' to receiving money 
Table 1 Participant characteristics of IPV survivors in Mexico City presenting to study clinics (total sample and subset that reported paid work in the year preceding the survey)

\begin{tabular}{|c|c|c|}
\hline & $\begin{array}{l}\text { Total sample that } \\
\text { derived latent } \\
\text { classes } \\
\mathrm{N}(\%)\end{array}$ & $\begin{array}{l}\text { Subset of total } \\
\text { sample that } \\
\text { worked in the } \\
\text { past year } \mathrm{n}(\%)\end{array}$ \\
\hline Overall & $947(100.0)$ & $572(60.4)$ \\
\hline Age, M (SD) & $29.86(7.16)$ & $30.71(7.23)$ \\
\hline No of children, M (SD) & $2.20(1.20)$ & $2.21(1.23)$ \\
\hline \multicolumn{3}{|l|}{ Birthplace } \\
\hline Mexico City & $677(71.5)$ & $414(72.4)$ \\
\hline State of Mexico, other republic or abroad & $270(28.5)$ & $158(27.6)$ \\
\hline $\begin{array}{l}\text { Previously screened for IPV in healthcare } \\
\text { setting }\end{array}$ & $95(10.0)$ & $64(11.2)$ \\
\hline \multicolumn{3}{|l|}{ Relationship status } \\
\hline Single & $57(6.0)$ & $43(4.5)$ \\
\hline Married & $227(23.9)$ & $151(26.4)$ \\
\hline Common-law marriage & $635(67.1)$ & $361(63.1)$ \\
\hline Separated and/or divorced & $28(2.9)$ & $17(2.9)$ \\
\hline \multicolumn{3}{|l|}{ Reason for healthcare visit ${ }^{*}$} \\
\hline General consultation & $232(24.6)$ & $150(26.3)$ \\
\hline Gynaecology & $70(7.4)$ & $44(7.7)$ \\
\hline Other services & $643(68.0)$ & $377(66.0)$ \\
\hline \multicolumn{3}{|l|}{ Work status } \\
\hline Own personal or help family business & $59(6.2)$ & $59(10.3)$ \\
\hline Selling/making products & $166(17.5)$ & $166(29.0)$ \\
\hline Animal husbandry or fieldwork & $2(0.2)$ & $2(0.3)$ \\
\hline Students & $11(1.2)$ & $11(1.9)$ \\
\hline Multiple informal jobs & $163(17.2)$ & $163(28.5)$ \\
\hline Employed, non-specified category & $171(18.1)$ & $171(29.9)$ \\
\hline Housewife (no paid work) & $357(37.7)$ & NA \\
\hline Looking for work (no paid work) & $18(1.9)$ & NA \\
\hline \multicolumn{3}{|l|}{ Education } \\
\hline No schooling & $26(2.8)$ & $18(3.2)$ \\
\hline Primary and secondary & $604(63.8)$ & $359(62.8)$ \\
\hline High school and beyond & $317(33.5)$ & $195(34.1)$ \\
\hline \multicolumn{3}{|l|}{ Physical IPV } \\
\hline Pushed and shoved & $869(91.8)$ & $529(92.9)$ \\
\hline Twisted and kicked & $423(44.7)$ & $258(45.1)$ \\
\hline Hit or thrown object & $251(26.5)$ & $165(28.9)$ \\
\hline Burned and threats of weapon use & $86(9.1)$ & $55(9.6)$ \\
\hline Hit with fist & $434(45.8)$ & $265(46.3)$ \\
\hline Choked or strangled & $283(29.9)$ & $175(30.6)$ \\
\hline \multicolumn{3}{|l|}{ Sexual IPV } \\
\hline Forced sex by threats & $207(21.9)$ & $125(21.9)$ \\
\hline Sex by intimidation & $279(29.5)$ & $176(30.8)$ \\
\hline Sex by physical force & $148(15.6)$ & $103(18.0)$ \\
\hline \multicolumn{3}{|l|}{ Injuries } \\
\hline Broken bones and teeth & $56(5.9)$ & $37(6.5)$ \\
\hline Burns and stitches & $50(5.3)$ & $35(6.1)$ \\
\hline Bruises & $592(62.5)$ & $364(63.6)$ \\
\hline Passed out & $72(7.6)$ & $47(8.2)$ \\
\hline Aches and pains & $494(52.2)$ & $307(53.7)$ \\
\hline Work-related disruptions & NA & $232(40.6)$ \\
\hline
\end{tabular}

*Values are given as number (column percentage). Column percentages are rounded to nearest tenth and may not equal 100 .

†Two cases missing on data for reason for visit and therefore not included in the table. IPV, intimate partner violence; $M$, mean; NA, not applicable. or income for their activities in the formal and informal sectors were included in the analysis.

\section{Data analysis}

We performed descriptive statistics of types of IPV, demographics and the outcomes. All analyses were conducted using SAS V.9.4 (SAS Institute, Cary, North Carolina, USA).

Next, we conducted a series of latent class models using the 14 IPV indicators. Each model's fit was assessed using the likelihood ratio test $G^{2}$, the Akaike information criterion (AIC) and the adjusted Bayesian information criterion (ABIC). Smallest values for AIC and ABIC, and a non-significant $P$ value for $G^{2}$ suggest better model fit. ${ }^{21}$ We also evaluated the entropy and model identification was assessed by running the models with 1000 randomly selected starting values and reviewing the percentage of times the model converged at the highest log-likelihood value. An entropy equal to or greater than 0.80 suggest adequate classification properties of the model. ${ }^{32}$ PROC LCA and LTA add-on software to SAS V.9.4 (SAS Institute) was used to fit the latent class models while accounting for clustering. ${ }^{33}$ The names of the latent classes were based on the probability of the indicators relative to 0.50 (ie, high $>0.50$, low $<0.50$ ). ${ }^{21}$

Separate multilevel risk regression analyses were used to examine unadjusted and adjusted models testing the effects of latent class membership on paid work disruption. We used multilevel modelling to address clustering in the data (ie, individuals were nested within health clinics). The generalised mixed model in GENMOD procedure was used to fit the multilevel models with a Poisson distribution for optimising precision. ${ }^{34}$ Each risk regression model adjusted for age, number of children, birthplace, occupation, IPV screening, educational status, relationship status and reason for healthcare visit. Relative risk (RR), 95\% CIs and $\mathrm{P}$ values < 0.05 were used to assess significance. These analyses were restricted to women who reported working, resulting in a subsample of 572 .

\section{RESULTS}

\section{Participant characteristics}

On average, participants who worked in the past year were 30 years old (SD 7.23 years) and had two children (SD 1.23 children) (table 1). A total of $72.4 \%$ were born in Mexico City, and most were in a common law marriage (63.1\%) or married (26.4\%). Further details are in table 1.

\section{Identifying latent classes based on IPV experiences}

IPV experiences for the entire sample are summarised in table 1. The LCA suggest four distinct classes based on experiences of IPV (figure 1). The four-class model was chosen because the $\mathrm{P}$ value for $\mathrm{G}^{2}$ was non-significant, and the AIC and ABIC test statistics were smallest. The entropy for the four-class model suggests that women were appropriately classified in their groups approximately $80 \%$ of the time. The four-class model was well defined, with $100.0 \%$ random sets producing the maximum-likelihood solution. Classes were named based on the proportion of violence experienced among women in their respective class. The Low Physical and Sexual IPV and Low Injuries class was the largest class accounting for $39.1 \%$ of the sample and comprised women reporting a low prevalence of physical and sexual IPV and injuries. The High Physical and Low Sexual IPV and Injuries class was the second largest class accounting for 36.5\% of the sample and comprised women reporting a high prevalence of physical IPV and injuries and a low prevalence of sexual IPV. The High Physical and Sexual IPV and Injuries class was the third 


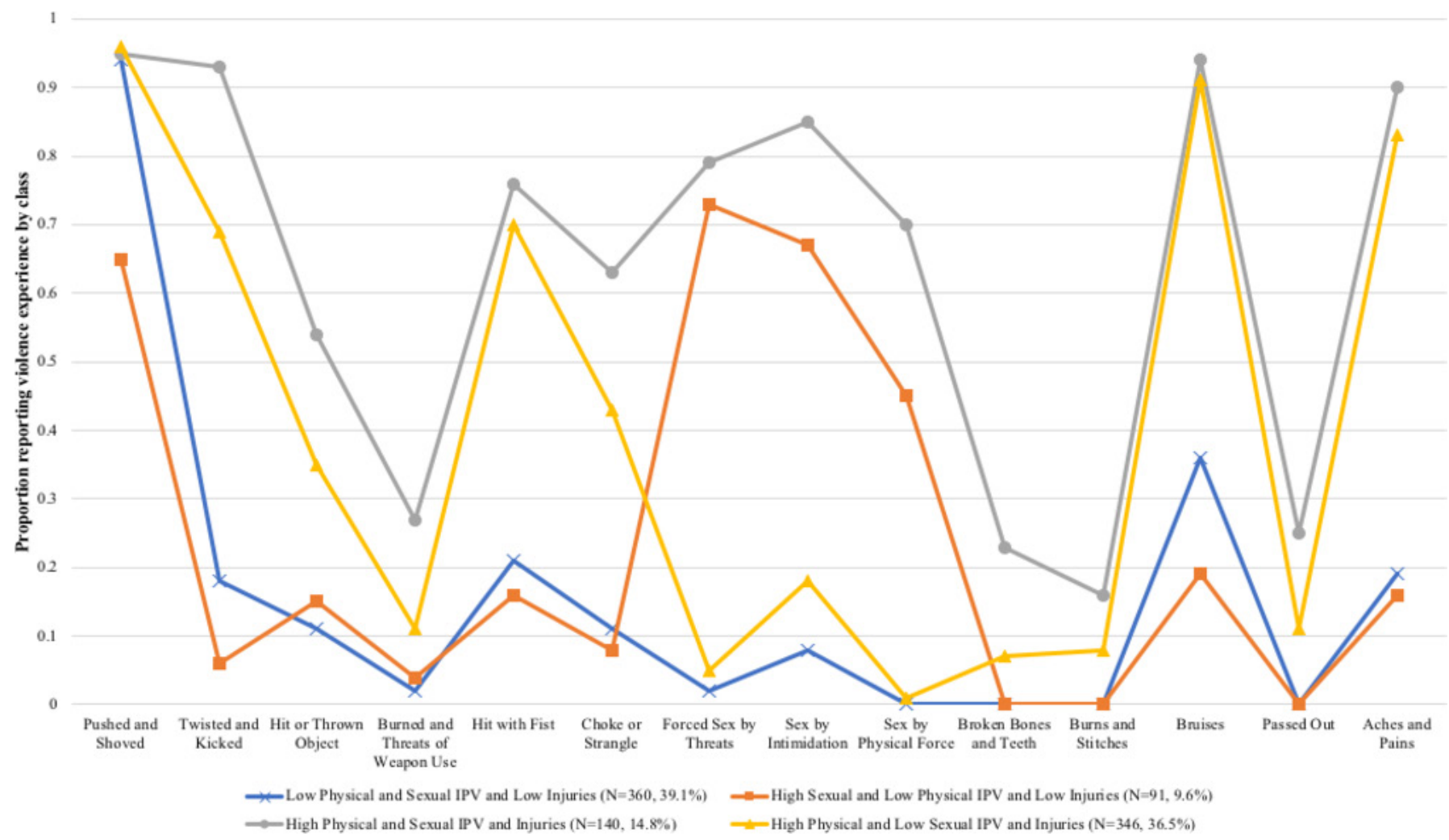

Figure 1 Forms and frequencies of physical and sexual intimate partner violence by latent class. IPV, intimate partner violence. The colour version of this figure is available online.

class accounting for $14.8 \%$ of the sample and comprised women reporting a high prevalence of physical and sexual IPV and injuries. The High Sexual and Low Physical IPV and Low Injuries class was the smallest class accounting for $9.6 \%$ of the sample and comprised women reporting a high prevalence of sexual IPV and a low prevalence of physical IPV and injuries.

\section{Associations between latent class membership and paid work disruptions due to IPV}

Latent class membership was associated with work-related disruptions (table 2). The unadjusted analysis suggests that the risk of work-related disruptions was significantly higher among women in the High Physical and Sexual IPV and Injuries (RR 2.64, 95\% CI 2.00 to 3.50) and women in the High Sexual and Low Physical IPV and Low Injuries (RR 2.08, 95\% CI 1.58 to 2.72) compared with women in the Low Physical and Sexual IPV and Low Injuries class. Latent class membership in the High Physical and Sexual IPV and Injuries (adjusted relative risk (ARR) 2.44, 95\% CI 1.80 to $3.29, \mathrm{P}<0.001$ ) and women in the High Sexual and Low Physical IPV and Low Injuries (ARR 2.05, $95 \%$ CI 1.56 to $2.70, \mathrm{P}<0.001$ ) remained significantly associated with violence disrupting work after adjustment.

\section{DISCUSSION}

This clinic-based study found a high level of work-related disruption due to IPV among low-income women in Mexico City with recent IPV experiences. One in four women reported either having to change jobs, miss work or school, or losing their job due to the abuse they were experiencing. The findings underscore how IPV can impede engagement in paid work among IPV survivors and thus provides support for conceptualising IPV as a global economic and workplace health issue, in addition to a critical public health and human rights issue.
Four distinct classes of IPV experiences were identified. Few studies have examined patterns of IPV experiences within Mexico or other LMICs, thus direct comparisons are difficult. Nonetheless, findings are consistent with other work that underscores how the emergence of distinct classes and the use of using LCA to improve understanding of heterogeneity of IPV experiences among vulnerable women. ${ }^{2435}$ For instance, while all women in the study sample had reported either physical or sexual violence during the year preceding the survey, neither levels of specific forms of IPV nor levels of injuries nor specific forms of violence were consistently experienced. Furthermore, high levels of injuries were only present in two classes (High Physical/Sexual IPV and Injuries; High Physical/Low Sexual IPV and Injuries), where there such injuries also occurred in combination with high levels of physical IPV. When high levels of sexual IPV were experienced, as in the High Sexual and Low Physical IPV class, low levels of both physical IPV and injury were also present; this class was also the smallest of the four classes. Notably, the largest class was the Low Physical IPV/Low Sexual IPV/Low Injuries class, followed by the High Physical IPV/ Low Sexual IPV/Injuries class.

Importantly, latent classes of IPV experiences were differentially associated with work disruption. Women in the High Physical IPV/High Sexual IPV and Injuries had the greatest likelihood for reporting work-related disruptions in comparison to the Low Physical/Sexual IPV/Low Injuries class. Similarly, women in the High Physical/Low Sexual/Injuries class also had a greater likelihood for disruption, while the High Sexual/Low Physical/No Injuries class did not significantly differ in likelihood of work-related disruptions in comparison to the low IPV class. These findings suggest that work-related disruptions may be largely driven by the occurrence of injuries, in combination with high levels of other forms of IPV (physical and sexual). The presence of such injuries may disrupt work due to pain from violence severity 
Table 2 Unadjusted and adjusted relative risk regression analyses of latent classes of IPV and work-related disruptions $(n=572)$

\begin{tabular}{|c|c|c|}
\hline & RR $(95 \% \mathrm{CI})$ & ARR $(95 \% \mathrm{CI})$ \\
\hline \multicolumn{3}{|l|}{ Latent classes } \\
\hline $\begin{array}{l}\text { High Physical/Sexual IPV } \\
\text { and Injuries }\end{array}$ & $2.64(2.00 \text { to } 3.50)^{* * *}$ & $2.44(1.80 \text { to } 3.29)^{* * *}$ \\
\hline $\begin{array}{l}\text { High Physical/Low Sexual } \\
\text { IPV and Injuries }\end{array}$ & $2.08(1.58 \text { to } 2.72)^{* * *}$ & $2.05(1.56 \text { to } 2.70)^{* * *}$ \\
\hline $\begin{array}{l}\text { Low Physical and Sexual } \\
\text { IPV }\end{array}$ & 1.00 & 1.00 \\
\hline $\begin{array}{l}\text { High Sexual and Low } \\
\text { Physical IPV }\end{array}$ & $1.03(0.61$ to 1.75$)$ & $0.92(0.55$ to 1.56$)$ \\
\hline Age & $0.98(0.97 \text { to } 0.99)^{* *}$ & $1.02(1.01 \text { to } 1.03)^{* *}$ \\
\hline No of children & 0.98 (0.92 to 1.03$)$ & 0.93 (0.86 to 1.02$)$ \\
\hline \multicolumn{3}{|l|}{ Birthplace } \\
\hline Mexico City & 1.04 (0.89 to 1.23$)$ & 0.94 (0.76 to 1.16$)$ \\
\hline $\begin{array}{l}\text { State of Mexico, other } \\
\text { republic or abroad }\end{array}$ & 1.00 & 1.00 \\
\hline $\begin{array}{l}\text { Previously screened for IPV in } \\
\text { healthcare setting }\end{array}$ & $1.40(1.05 \text { to } 1.87)^{*}$ & 1.11 (0.87 to 1.42$)$ \\
\hline \multicolumn{3}{|l|}{ Relationship status } \\
\hline Single & 0.99 (0.77 to 1.28$)$ & $0.99(0.70$ to 1.40$)$ \\
\hline Married & $0.96(0.82$ to 1.13$)$ & $0.95(0.78$ to 1.20$)$ \\
\hline Common-law marriage & 1.00 & 1.00 \\
\hline Separated and divorced & 2.06 (0.98 to 4.33$)$ & 1.38 (0.97 to 1.97$)$ \\
\hline \multicolumn{3}{|l|}{ Reason for healthcare visit } \\
\hline General consultation & 0.88 (0.75 to 1.04$)$ & 1.10 (0.90 to 1.35$)$ \\
\hline Gynaecology & 1.04 (0.82 to 1.32$)$ & 1.03 (0.67 to 1.57$)$ \\
\hline Other services & 1.00 & 1.00 \\
\hline \multicolumn{3}{|l|}{ Occupation } \\
\hline Selling/making products & $0.74(0.56 \text { to } 0.96)^{*}$ & 0.78 (0.61 to 1.01$)$ \\
\hline Multiple informal jobs & 1.02 (0.81 to 1.29$)$ & 0.99 (0.79 to 1.23$)$ \\
\hline $\begin{array}{l}\text { Employed (type not } \\
\text { specified) }\end{array}$ & 1.00 & 1.00 \\
\hline $\begin{array}{l}\text { Other (personal/family } \\
\text { business, fieldwork, } \\
\text { students) }\end{array}$ & 0.70 (0.48 to 1.02$)$ & 0.82 (0.57 to 1.18$)$ \\
\hline \multicolumn{3}{|l|}{ Education } \\
\hline No schooling & 1.00 & 1.00 \\
\hline Primary and secondary & 0.54 (0.23 to 1.28$)$ & 2.19 (0.88 to 1.57$)$ \\
\hline High school and beyond & $0.64(0.27$ to 1.55$)$ & 1.90 (0.77 to 1.59$)$ \\
\hline
\end{tabular}

and/or shame if the injuries are visible, as shame from injuries has been underscored in other studies. ${ }^{183637}$ Furthermore, the presence of such injuries that occur in combination with high levels of physical and sexual IPV may also be a marker for severe IPV that poses the most direct threat to a woman's safety and autonomy in other spheres, such as reproductive health, ${ }^{38}$ and thus may extend to higher levels of disruptions in paid work. Additional research is needed to explore such potential mechanisms, including injuries as a mediator, and to better understand why class 4 (High Sexual IPV/Low Physical IPV/No Injuries) did not significantly relate to increased work-related disruptions. It may be that the negative health impacts of belonging to this class may manifest with other health outcomes, such as mental health or reproductive health.

The current study has limitations. First, while the job-related disruptions specifically asked about whether disruptions occurred because of IPV, participants may have responded in the affirmative based on non-IPV-related factors. Also, the work-related disruption outcome was inclusive of one item that also included school-related disruptions. However, only two women identified as students and responded in the affirmative to the item that included school-related issues. The LCA classes are statistical constructs, thus additional research, including qualitative research, is needed. The analysis used a clinic-based sample composed of low-income women in Mexico City, with recent IPV experiences, who met specific criteria for inclusion into an RCT. Thus, the latent classes may be only generalisable to similar demographics reported herein. It is also possible that women may have experienced work-related disruptions due to IPV that occurred prior to the past 12 months. Lastly, despite attention to ethical and privacy concerns, IPV experiences may be under-reported, and this may influence the classes.

These limitations notwithstanding, the current study has several implications for programming and future research. Given the high levels of paid work disruption due to IPV in this clinic-based study, the health sector may seek to integrate work-related disruptions into ongoing responses to IPV. The high frequency of women who reported earning money through informal sectors also highlights the importance of outreach to women engaged in informal sector employment regarding IPV safety planning, outreach regarding resources and prevention interventions. Study findings herein strongly underscore the importance of understanding typologies of IPV experiences as a step towards informing more tailored programming. Future research is also needed to clarify underlying mechanisms through which the different patterns of IPV experiences impact work-related disruptions to better inform potential interventions, with a particular focus on injuries.

Furthermore, findings are consistent with existing research on the importance of addressing gender norms and IPV in economic empowerment programming, and thus underscores

\section{What is already known on this subject}

- Women's employment status has been widely document as a risk factor for experiencing intimate partner violence. In high-income countries, research has also documented how intimate partner violence impacts women's work outcomes (eg, job instability, absenteeism, lower productivity). Very little is known, however, how intimate partner violence and which specific patterns of intimate partner violence experiences impact women's work outcomes in low-income and middle-income countries, such as Mexico.

\section{What this study adds}

- In Mexico City, a high frequency of work-related disruption due to intimate partner violence was documented among a clinic-based sample of low-income women who were survivors of intimate partner violence. Through latent class analysis, distinct patterns of violence experiences were identified. These patterns of intimate partner violence were also related to work disruption. This was one of the first studies to investigate basic patterning of intimate partner violence experiences and how such patterns relate to women's work outcomes in a low-income and middleincome country. Study findings highlight the importance of considering women's safety within work settings and, more broadly, economic development programming. 
the importance of economic empowerment as a strategy for IPV prevention as well as reducing IPV to ensure economic development. ${ }^{39} 40$

Acknowledgements The authors thank the Mexico City Ministry of Health and MEXFAM for their collaboration. The authors would also like to thank Helena Acosta from International Planned Parenthood International for training the nurses in our study. The authors would also like to thank the women and nurses who participated in this study.

Contributors JG had full access to all of the data in the study and takes responsibility for the integrity of the data and the accuracy of the data analysis. Study concept and design: JG, TCW, CDO, CGM. Acquisition of data: JG, KLF, PAC, CDO. Analysis and interpretation of data: JG, TCW, KLF, PAC, CH, CDO, CGM, CAO. Drafting of the manuscript: JG, TCW. Critical revision of the manuscript for important intellectual content: JG, TCW, KLF, PAC, CH, CDO, CGM, CAO. Statistical analysis: TCW, JG. Obtained funding: JG, CDO. Administrative, technical or material support: $J G, K L F, P A C, C D O$

Funding The study was funded by an anonymous donor administered by the Vanguard Charitable Endowment Program. Based on the stipulations set forth by the donor, we are not permitted to disclose the funder (PI: JG). Partial support was also provided by NIMH to Yale Center for Interdisciplinary Research on AIDS (T32MH020031).

Disclaimer The views expressed herein are solely those of the authors and do not necessarily represent the views, policies, or decisions of the $\mathrm{NIH}$, NIMH, the $\mathrm{MOH}$, or the World Health Organization.

Competing interests None declared.

Patient consent Obtained.

Ethics approval All study protocols were approved by Yale School of Public Health (no. 1202007993), George Mason University (protocol no. 704016-4), Innovations for Poverty Action (no. 00006083) and the Mexico City Ministry of Health (protocol no. 1470-6812) human subjects committees.

Provenance and peer review Not commissioned; externally peer reviewed.

Data sharing statement Due to the sensitive nature of the data, the data cannot be made publicly available. Requests for data should be sent to JG.

Open access This is an Open Access article distributed in accordance with the Creative Commons Attribution Non Commercial (CC BY-NC 4.0) license, which permits others to distribute, remix, adapt, build upon this work non-commercially, and license their derivative works on different terms, provided the original work is properly cited and the use is non-commercial. See: http://creativecommons.org/ licenses/by-nc/4.0/

(c) Article author(s) (or their employer(s) unless otherwise stated in the text of the article) 2018. All rights reserved. No commercial use is permitted unless otherwise expressly granted.

\section{REFERENCES}

1 World Health Organization. Global and regional estimates of violence against women: prevalence and health effects of intimate partner violence and non-partner sexual violence. Geneva: World Health Organization, 2013.

2 Staggs SL, Riger S. Effects of intimate partner violence on low-income women's health and employment. Am J Community Psychol 2005;36:133-45.

3 Crowne SS, Juon HS, Ensminger M, et al. Concurrent and long-term impact of intimate partner violence on employment stability. I Interpers Violence 2011;26:1282-304.

4 Borchers A, Lee RC, Martsolf DS, et al. Employment maintenance and intimate partner violence. Workplace Health Saf 2016:469-78.

5 Swanberg JE, Logan T, Macke C. Intimate partner violence, employment, and the workplace: consequences and future directions. Trauma Violence Abuse 2005;6:286-312.

6 Tiesman HM, Gurka KK, Konda S, et al. Workplace homicides among U.S. women: the role of intimate partner violence. Ann Epidemiol 2012;22:277-84.

7 Vyas S, Heise L. Using propensity score matching to estimate an "unbiased effectsize" between women's employment and partner violence in Tanzania. J Interpers Violence 2014;29:2971-90.

8 Vyas S, Heise L. How do area-level socioeconomic status and gender norms affect partner violence against women? Evidence from Tanzania. Int J Public Health 2016;61:971-80.

9 Vyas $\mathrm{S}$, Jansen HA, Heise L, et al. Exploring the association between women's access to economic resources and intimate partner violence in Dar es Salaam and Mbeya, Tanzania. Soc Sci Med 2015;146:307-15.

10 Krishnan S, Rocca CH, Hubbard AE, et al. Do changes in spousal employment status lead to domestic violence? Insights from a prospective study in Bangalore, India. Soc Sci Med 2010;70:136-43.
11 Ajala EM. Impact of domestic violence on the workplace and workers' productivity in selected industries in Nigeria. The Anthropologist 2008;10:257-64.

12 GEOGRAFÍA INDEY. Encuesta Nacional sobre la Dinámica de las Relaciones en los Hogares (2011). Panorama de violencia contra las mujeres en Estados Unidos Mexicanos. Mexico: INEGI, 2013.

13 Ávila-Burgos L, Valdez-Santiagob R, Barroso-Quiab A, et al. [Prevalence and factors associated with intimate partner abuse in female users of public health services in Mexico: a comparative analyses]. Rev Invest Clin 2014;66:45-58.

14 Terrazas-Carrillo EC, McWhirter PT. Employment status and intimate partner violence among Mexican women. J Interpers Violence 2015;30:1128-52.

15 Valdez-Santiago R, Hijar M, Rojas Martínez R, et al. Prevalence and severity of intimate partner violence in women living in eight indigenous regions of Mexico. Soc Sci Med 2013;82:51-7.

16 (OECD) TOfEC-oaD. Closing the Gender Gap: Act Now Mexico. OECD. 2012 http:// www.oecd.org/gender/Closing\%20the\%20Gender\%20Gap\%20-\%20Mexico\% 20FINAL.pdf.

17 Garcia-Moreno C, Jansen H, Ellsberg M, et al. WHO multi-country study on women's health and domestic violence against women: initial results on prevalence, health outcomes and women's responses. Geneva: World Health Organization, 2005.

18 Campbell J, Jones AS, Dienemann J, et al. Intimate partner violence and physical health consequences. Arch Intern Med 2002;162:1157-63.

19 Coker AL, Davis KE, Arias I, et al. Physical and mental health effects of intimate partner violence for men and women. Am J Prev Med 2002;23:260-8.

20 Hebenstreit CL, Maguen S, Koo KH, et al. Latent profiles of PTSD symptoms in women exposed to intimate partner violence. J Affect Disord 2015;180:122-8.

21 Collins LM, Lanza ST. Latent class and latent transition analysis with applications in the social, behavioral, and health sciences. Hoboken, NJ: John Wiles \& Sons, 2010.

22 Golder S, Connell CM, Sullivan TP. Psychological distress and substance use among community-recruited women currently victimized by intimate partners: a latent class analysis and examination of between-class differences. Violence Against Women 2012;18:934-57.

23 Young-Wolff KC, Hellmuth J, Jaquier $\mathrm{V}$, et al. Patterns of resource utilization and mental health symptoms among women exposed to multiple types of victimization: a latent class analysis. J Interpers Violence 2013;28:3059-83.

24 Sipsma HL, Falb KL, Willie T, et al. Violence against Congolese refugee women in Rwanda and mental health: a cross-sectional study using latent class analysis. BMJ Open 2015:5:e006299.

25 United Nations. Transforming Our World: the 2030 Agenda for Sustainable Development. New York: United Nations, 2015.

26 Gupta J, Falb KL, Ponta O, et al. A nurse-delivered, clinic-based intervention to address intimate partner violence among low-income women in Mexico City: findings from a cluster randomized controlled trial. BMC Med 2017;15:128.

27 Falb KL, Diaz-Olavarrieta C, Campos PA, et al. Evaluating a health care provider delivered intervention to reduce intimate partner violence and mitigate associated health risks: study protocol for a randomized controlled trial in Mexico City. BMC Public Health 2014;14:772.

28 Agenda estadistica. 2008 http://www.salud.df.gob.mx/ssdf/media/Agenda2008/.

29 Bott SGA, Claramunt MC, Guezmes A. IPPF/WHR, ed. Improving the health sector response to gender based violence: a resource manual for health care professionals in developing countries. New York, NY: IPPF/WHR, 2010

30 Díaz-Olavarrieta C, Ellertson C, Paz F, et al. Prevalence of battering among 1780 outpatients at an internal medicine institution in Mexico. Soc Sci Med 2002;55:1589-602.

31 World Health Organization. WHO multi-country study on women's health and domestic violence against women: summary report of initial results on prevalence, health outcomes and women's responses. Geneva: World Health Organization, 2005.

32 McCutcheon AL. Latent class analysis. New York, NY: Sage Publications, 1987.

33 Lanza ST, Dziak J, Huang L, et al. PROC LCA \& PROC LTA users' guide (version 1.3.0): University Park: The Methodology Center, 2014

34 Spiegelman D, Hertzmark E. Easy SAS calculations for risk or prevalence ratios and differences. Am J Epidemiol 2005;162:199-200.

35 Sullivan TP, Holt LJ. PTSD symptom clusters are differentially related to substance use among community women exposed to intimate partner violence. J Trauma Stress 2008:21:173-80.

36 Shuman SJ, Falb KL, Cardoso LF, et al. Perceptions and experiences of intimate partner violence in Abidjan, Côte d'Ivoire. PLoS One 2016;11:e0157348.

37 Hegarty KL, O'Doherty LJ, Chondros P, et al. Effect of type and severity of intimate partner violence on women's health and service use: findings from a primary care trial of women afraid of their partners. J Interpers Violence 2013;28:273-94.

38 Rahman M, Nakamura K, Seino K, et al. Intimate partner violence and use of reproductive health services among married women: evidence from a national Bangladeshi sample. BMC Public Health 2012;12:913.

39 Gupta J, Falb KL, Lehmann H, et al. Gender norms and economic empowerment intervention to reduce intimate partner violence against women in rural Côte d'Ivoire: a randomized controlled pilot study. BMC Int Health Hum Rights 2013;13:46.

40 Pronyk PM, Kim JC, Abramsky T, et al. A combined microfinance and training intervention can reduce HIV risk behaviour in young female participants. AIDS 2008;22:1659-65 